\title{
Medication-Related Quality of Life in Thai Epilepsy Patients
}

Original Article

Journal of Epilepsy Research pISSN 2233-6249 / elSSN 2233-6257

Received November 1, 2019

Revised January 2, 2020

Accepted January 31, 2020

Corresponding author:

Phantipa Sakthong, $\mathrm{PhD}$

Department of Pharmacy Practice, Faculty of Pharmaceutical Sciences, Chulalongkorn University, Phyathai Road, Pathumwan, Bangkok 10330, Thailand

Tel. +66-22188408

Fax. +66-22188403

E-mail; phantipa.s@pharm.chula.ac.th

\author{
Phantipa Sakthong, PhD, Bavornpat Suriyapakorn, Pharm D \\ Department of Pharmacy Practice, Faculty of Pharmaceutical Sciences, Chulalongkorn University, Bangkok, \\ Thailand
}

Background and Purpose: This study aimed to assess medication-related quality of life in Thai patients with epilepsy. The second objective was to evaluate the associations between the medication therapy-related quality of life and patient characteristics.

Methods: This was a cross-sectional study. A convenience sample of 173 outpatients with epilepsy was recruited from a university hospital in Bangkok, Thailand. Inclusion criteria were aged 18 or over who were continuously taking an epileptic drug for at least 3 months, understanding Thai language, and willing to participate in the study. The Patient-Reported Outcomes Measure of Pharmaceutical Therapy for Quality of Life (PROMPT-QoL) was utilized to measure the medication therapy-related quality of life. Pearson's correlation coefficients and stepwise multiple linear regressions were employed to assess the relationships between eight PROMPT-QoL domain scores and patient demographic and clinical characteristics.

Results: Mean age was $36.4 \pm 9.5$ years and approximately $57 \%$ were female. Among eight domains of the PROMPT-QoL, the therapeutic relationships with health care providers and psychological impacts of medication use domains yielded the highest (77.9) and lowest (61.9) mean scores, respectively. Seven out of eight PROMPT-QoL domain scores were considered as moderate-to-good. Age, gender, duration of epilepsy, seizure frequency, and treatment preference were significantly associated with PROMPT-QoL domain scores in multivariate linear regression analyses.

Conclusions: This study showed that Thai patients with epilepsy had moderate-to-good medication therapy-related quality of life. Healthcare providers should pay more attention to patients' psychological impacts of antiepileptic drugs and those with characteristics related to lower medication therapy-related quality of life. (2019;9:139-146)

Key words: Epilepsy, Medication, Drug, Pharmaceutical, Quality of life, Thailand

\section{Introduction}

Epilepsy is a common chronic neurological disease that affects around 50 million people worldwide and $80 \%$ of people with epilepsy live in low- and middle-income countries. ${ }^{1}$ In rural Thailand, its prevalence was estimated to be 7.2 per 1,000 people. ${ }^{2}$

Epilepsy is defined as having two or more unprovoked seizures separated by 24 hours. ${ }^{3}$ Epilepsy can have an extremely negative impact on patients' quality of life due to seizure frequency and adverse effects of antiepileptic drugs. ${ }^{4-6}$ Nevertheless, up to $70 \%$ of people living with epilepsy could live seizure-free with appropriate use of antiepileptic drugs. ${ }^{1}$ Therefore, antiepileptic drugs can have both pos- itive and negative effects on patients' lives, so assessment medication-related quality of life in patients with epilepsy is essential.

One of the most widely used quality of life questionnaires in patients with epilepsy is the Quality of Life in Epilepsy (QOLIE-31) which is an epilepsy-specific measure. ${ }^{7}$ However, the QOLIE-31 focuses on measuring the impacts of epilepsy on patients' lives rather than those of medication use since there are only three out of 31 items assessing the impacts of medication. Other tools are also related to measuring the side effects of antiepileptic drugs. ${ }^{6}$ Hence, previous studies in this area have not extensively assessed medication-related quality of life in patients with epilepsy. ${ }^{4-6}$

A novel generic questionnaire called Patient-Reported Outcomes 
Measure of Pharmaceutical Therapy for Quality of Life (PROMPT-QoL) was developed. ${ }^{8}$ It was originally in Thai and was mainly created by applying the concept of patient-centered pharmaceutical care. ${ }^{9}$ In this concept, a practitioner has to be responsible for patients' drug-related needs including drug and disease understanding, expectation of effectiveness, safety or concern, and convenience to achieve their positive outcomes. The PROMPT-QoL can also be used to measure the impacts of medication therapy on patient's physical, psychological, and social aspects, etcetera. The PROMPT-QoL was found to be practical, reliable, valid, ${ }^{10}$ and responsive ${ }^{11}$ in Thai patients with chronic diseases. Thus, this study aimed to assess medication-related quality of life using the PROMPT-QoL, a specific medication measure for health-relate quality of life, in Thai patients with epilepsy. Little was also known about associations between medication-related quality of life and patient characteristics in epilepsy patients since most previous studies assessed the relationships with health-related quality of life, non-specific medication measures. ${ }^{4-6,12-15}$ Hence, the second objective was to evaluate such associations. This information will be helpful for healthcare providers to manage medication therapy for epilepsy patients.

\section{Methods}

\section{Participants and procedures}

This study was a cross-sectional survey. A convenience sampling was employed to recruit patients with epilepsy from King Chulalongkorn Memorial Hospital, a university hospital, in Bangkok, Thailand, between October 2016 and March 2017. Outpatients aged 18 or over, who were continuously taking an epileptic drug for at least 3 months, understood Thai language, and were willing to participate in the study, were included. Patients who could not complete the questionnaire were excluded. While waiting to see a physician at the hospital, the patients were asked to do the PROMPT-QoL by themselves. The data of patient characteristics including socio-demographic and clinical data were collected from interviews and medical records. Written informed consent was obtained from all patients. The study was approved by the Ethical Committee of the hospital (certificate of approval number: 812/2016).

\section{Measure}

\section{PROMPT-QoL}

The PROMPT-QoL has 43 items including nine domains: general attitude toward medication use (1 item), medication and disease in- formation (9 items), satisfaction with medication effectiveness (3 items), impacts of medications and side-effects (8 items), psychological impacts of medication use (9 items), convenience (3 items), availability and accessibility (4 items), therapeutic relationships with healthcare providers ( 3 items), and overall quality of life ( 3 items). Each item has 5-point Likert scale from "not at all" to "very much" except only one item of general attitude toward medication use domain, which provides respondents with four treatment types (medications, alternative medications [e.g., herbs, supplementary food, massage, acupuncture, mind-body interventions], both, or others than mentioned), and asks them to indicate their preferred type of treatment. Thus, only 42 items are summed to calculate the other eight PROMPT-QoL domain scores. The recall period of the PROMPT-QoL is today. In this study, the Cronbach's alpha of the 42-item PROMPT-QoL was 0.923, which was interpreted as good internal consistency reliability. ${ }^{16}$

Item scores range from 1 to 5 , with higher scores indicating better quality of life. Observed domain scores are then converted to percentages (0-100) using the following formula: Domain scores $=100 \times$ (observed score - minimum domain score) / (maximum domain score - minimum domain score). Domain scores range between 0-25, 26-50, 51-75, and 76-100, which are interpreted as low, fair-to-moderate, moderate-to-good and good-to-excellent quality of life, respectively.

\section{Data analysis}

To summarize patient characteristics, means, standard deviations, medians, and interquartiles were calculated for continuous variables, while frequencies and percentages were used for categorical variables. Descriptive statistics of the PROMPT-QoL were presented with means, standard deviations, medians and interquartiles of its items and domains. The general attitude toward medication use item was presented with percentages. For univarite analyses, Pearson's correlation coefficients were utilized to assess the relationships between eight PROMPT-QoL domain scores and patient characteristics. To adjust for confounders, stepwise multiple linear regressions were employed. The data were checked and met all assumptions for a multiple linear regression. The patient characteristics included age, gender, education, employment status, duration of epilepsy, number of antiepileptic drugs, seizure frequency per three months, and treatment preference. A rule of thumb of 10 to 12 participants per variable was used. ${ }^{17}$ Thus, the required sample size was at least 80 to fit multiple linear regression models. $p$-values less than 0.05 were con- 
sidered significant. All analyses were performed using IBM SPSS version 22 (IBM Corp., Bangkok, Thailand).

\section{Results}

\section{Patient characteristics}

Of 180 eligible patients, seven patients who could not complete the PROMPT-QoL were excluded. Thus, there were 173 patients left for data analysis. Table 1 shows the patient characteristics. The mean age was $36.4 \pm 9.5$ years and approximately $57 \%$ were female. Most patients finished at least university. More than half of the sample was employed. The average duration of epilepsy was $17.8 \pm 9.5$ years. Most patients used polytherapy. About half of the sample had at least one seizure per three months.

Table 1. Patient characteristics $(n=173)$

\begin{tabular}{lc}
\hline Characteristic & Value \\
\hline Age (years) & $36.4 \pm 9.5$ \\
Median (interquartile) & $36(29-44)$ \\
Gender & \\
Male & $75(43.4)$ \\
Female & $98(56.6)$ \\
Number of education years & $12.5 \pm 3.8$ \\
Median (interquartile) & $12(9-16)$ \\
Employment status & \\
Employed & $97(56.1)$ \\
Housewife & $18(10.4)$ \\
Student & $12(6.9)$ \\
Retired & $7(4.1)$ \\
Unemployed & $39(22.5)$ \\
Duration of epilepsy (years) & $17.8 \pm 9.5$ \\
Median (interquartile) & $17(12.0-21.5)$ \\
Number of antiepileptic drugs & $2.2 \pm 1.1$ \\
Median (interquartile) & $2(2-3)$ \\
Seizure frequency per 3 months & \\
0 & $87(50.3)$ \\
Treatment preference & $86(49.6)$ \\
Medications & \\
Alternative medications & $132(76.3)$ \\
Both medications and alternative medications & $29(16.8)$ \\
Others & $8.6)$ \\
\hline & \\
\hline
\end{tabular}

Values are presented as mean \pm standard deviation or number (\%) unless otherwise indicated.

\section{Medication therapy-related quality of life}

For the general attitude toward medication use question, most of the sample preferred to use only medication treatment $(76 \%)$, while the others wanted both medication and alternative treatments $(17 \%)$, others $(5 \%)$, or only alternative treatments $(2 \%)$, respectively (Table 1). The descriptive statistics of the items and the other domains of the PROMPT-QoL are presented in Table 2.

For the medication and disease information domain, the items with the mean scores lower than 3.5 included "strength (3.2)" and "what to do if medication doses are missed (3.4)". For the satisfaction with medication effectiveness domain, the "onset of medication action" item provided the lowest mean score (3.7). For the impacts of medications and side-effects domain, the item "memory and cognition" yielded the lowest mean score (3.5). Regarding the psychological impacts of medication use domain, all items had the mean scores lower than 4 except only the item "taking medication in front of others" (4.2).

For the convenience domain, the item "appropriate dosage forms" provided the lowest mean score (3.5). For the availability and accessibility domain, the items "service process and waiting time" and "travel or self-support to hospital" yielded the lowest mean scores of 3.5. For the therapeutic relationships with healthcare providers domain, all items provided the mean scores above 4 . For the overall quality of life domain, the item "happiness" had the lowest mean score (3.6).

As for domain scores, the therapeutic relationships with health care providers and the psychological impacts of medication use domains yielded the highest (77.9) and lowest (61.9) mean scores, respectively.

\section{Associations between medication therapy-related quality of life and patient characteristics}

As shown in Table 3, younger age, shorter duration of epilepsy, and higher number of antiepileptic drugs were significantly associated with higher medication and disease information domain scores. However, after adjusting for covariates by the multiple linear regression (Table 4), only age was significantly correlated. Patients with free seizure and medication preference had significantly higher satisfaction of medication effectiveness domain scores than those with seizure and combined medication and alternative treatments (both univariate and multivariate analyses).

Patients with medication preference provided significantly higher impacts of medications and side-effects domain scores than those with combined medication and alternative treatments (both uni- 
Table 2. Descriptive statistics of item and domain scores of the PROMPT-QoL

\begin{tabular}{|c|c|c|c|}
\hline Measure & Mean \pm SD & Median & Interquartile \\
\hline Receiving drug and disease information & $66.5 \pm 19.1$ & 66.7 & $55.6-79.2$ \\
\hline Drug name & $3.7 \pm 1.0$ & 4 & $3-4$ \\
\hline Strength & $3.2 \pm 1.2$ & 4 & $2-4$ \\
\hline Indication & $3.8 \pm 0.9$ & 4 & $3-4$ \\
\hline How to use medications & $3.9 \pm 0.9$ & 4 & $3-5$ \\
\hline Reason for using medications regularly & $3.9 \pm 1.0$ & 4 & $3-5$ \\
\hline What to do if medication doses are missed & $3.4 \pm 1.2$ & 4 & $3-4$ \\
\hline Side-effects and management & $3.6 \pm 1.1$ & 4 & $3-4$ \\
\hline Causes and prevention & $3.8 \pm 1.0$ & 4 & $3-4$ \\
\hline Symptoms, severity, and treatment & $3.7 \pm 1.0$ & 4 & $3-4$ \\
\hline Satisfaction with medication effectiveness & $72.8 \pm 20.8$ & 75.0 & $58.3-91.7$ \\
\hline Symptom relief & $4.0 \pm 0.9$ & 4 & $4-5$ \\
\hline Cure at first time & $4.0 \pm 1.0$ & 4 & $3-5$ \\
\hline Onset of medication action & $3.7 \pm 1.0$ & 4 & $3-4$ \\
\hline Impacts of medications and side-effects on & $72.5 \pm 20.2$ & 75.0 & $59.4-87.5$ \\
\hline Mobility, energy, pain, and discomfort & $3.8 \pm 1.2$ & 4 & $3-5$ \\
\hline Sleep & $3.7 \pm 1.1$ & 4 & $3-5$ \\
\hline Memory and cognition & $3.5 \pm 1.1$ & 4 & $3-4$ \\
\hline Appearance or body skin & $4.0 \pm 1.1$ & 4 & $3-5$ \\
\hline Eating, digestion, or stool passing & $4.2 \pm 1.1$ & 4 & $4-5$ \\
\hline Vision, hearing, and speech & $4.0 \pm 1.1$ & 4 & $3-5$ \\
\hline Intercourse and sexual desire & $4.4 \pm 1.0$ & 5 & $4-5$ \\
\hline Daily activities or socializing with others & $3.8 \pm 1.3$ & 4 & $3-5$ \\
\hline Psychological impacts of medication use & $61.9 \pm 23.5$ & 61.1 & $45.8-80.6$ \\
\hline Medication side effects & $3.4 \pm 1.2$ & 3 & $2-4$ \\
\hline Feeling bored with taking medication every day & $3.5 \pm 1.2$ & 4 & $3-5$ \\
\hline Medication resistance or ineffectiveness & $3.5 \pm 1.3$ & 4 & $3-5$ \\
\hline Medication dependence & $3.0 \pm 1.5$ & 3 & $2-4.5$ \\
\hline Changing type/strength of medication & $3.5 \pm 1.3$ & 3 & $3-5$ \\
\hline Taking many medications & $3.3 \pm 1.3$ & 3 & $2-4.5$ \\
\hline Taking medication in front of others & $4.2 \pm 1.0$ & 5 & $4-5$ \\
\hline Medication interaction & $3.6 \pm 1.2$ & 4 & $3-5$ \\
\hline Taking medication makes you less healthy than person with the same age & $3.3 \pm 1.3$ & 3 & $2-4$ \\
\hline Convenience & $67.3 \pm 20.0$ & 66.7 & $50.0-83.3$ \\
\hline Appropriate dosage forms & $3.5 \pm 1.0$ & 3 & $3-4$ \\
\hline Convenience of use & $3.7 \pm 1.0$ & 4 & $3-4$ \\
\hline Ease of carrying medications around & $3.8 \pm 0.9$ & 4 & $3-5$ \\
\hline Availability/accessibility & $69.0 \pm 18.1$ & 68.9 & $56.3-81.3$ \\
\hline Medication availability in a setting & $4.4 \pm 1.0$ & 5 & $4-5$ \\
\hline Medication and travel expenses & $3.7 \pm 1.2$ & 4 & $3-5$ \\
\hline Service process and waiting time & $3.5 \pm 1.0$ & 3 & $3-4$ \\
\hline Travel or self-support to hospital & $3.5 \pm 1.1$ & 3 & $3-4$ \\
\hline Therapeutic relationships with health care providers & $77.9 \pm 16.8$ & 75.0 & $66.7-91.7$ \\
\hline Trust doctor's decision on medication treatment & $4.2 \pm 0.8$ & 4 & $4-5$ \\
\hline Friendly manners and willingness to answer medication queries & $4.1 \pm 0.9$ & 4 & $4-5$ \\
\hline Getting help to sort out medication-related problems or concerns & $4.0 \pm 0.8$ & 4 & $4-5$ \\
\hline Overall quality of life & $68.8 \pm 20.4$ & 66.7 & $58.3-83.3$ \\
\hline Satisfaction with medication use & $3.9 \pm 0.9$ & 4 & $3.5-5$ \\
\hline Happiness & $3.6 \pm 0.9$ & 4 & $3-4$ \\
\hline Improvement in daily life & $3.7 \pm 1.09$ & 4 & $3-4$ \\
\hline
\end{tabular}

PROMPT-QoL, Patient-Reported Outcomes Measure of Pharmaceutical Therapy for Quality of Life; SD, standard deviation. 
Table 3. Pearson's correlation coefficients between PROMPT-QoL domain scores and patient characteristics

\begin{tabular}{|c|c|c|c|c|c|c|c|c|}
\hline & MDI & SME & IMS & PIMU & CON & AA & TRHC & OQOL \\
\hline Age (years) & $-0.201 *$ & 0.146 & 0.082 & 0.092 & $0.190^{\dagger}$ & $0.202 *$ & 0.060 & $0.202 *$ \\
\hline Gender ( 1 , female; 2 , male) & -0.021 & 0.056 & 0.120 & 0.148 & 0.063 & 0.038 & 0.035 & 0.028 \\
\hline Number of education years & 0.018 & -0.029 & 0.051 & -0.045 & -0.050 & -0.127 & 0.013 & -0.012 \\
\hline $\begin{array}{l}\text { Employment status } \\
\text { (1, unemployed/housewife/student/retired; } \\
\text { 2, employed) }\end{array}$ & -0.049 & -0.066 & 0.087 & 0.083 & 0.010 & 0.095 & -0.050 & -0.022 \\
\hline Duration of epilepsy (years) & $-0.178^{\dagger}$ & 0.094 & 0.101 & $0.164^{\dagger}$ & 0.019 & 0.131 & 0.007 & 0.142 \\
\hline Number of antiepileptic drugs & $0.167^{\dagger}$ & -0.066 & -0.041 & 0.050 & -0.014 & 0.059 & 0.143 & -0.007 \\
\hline Seizure frequency per 3 months $(1,0 ; 2,>0)$ & 0.083 & $-0.256^{*}$ & -0.129 & $-0.153^{\dagger}$ & $-0.197^{*}$ & -0.104 & -0.051 & $-0.274 *$ \\
\hline $\begin{array}{l}\text { Treatment preference ( } 1 \text {, medications; } 2 \text {, both } \\
\text { medications and alternative medications, } \\
\text { alternative medications and others) }\end{array}$ & -0.125 & $-0.274^{\star}$ & $-0.246^{*}$ & $-0.247^{*}$ & -0.064 & $-0.153^{\dagger}$ & -0.069 & -0.281 * \\
\hline
\end{tabular}

PROMPT-QoL, Patient-Reported Outcomes Measure of Pharmaceutical Therapy for Quality of Life; MDI, medication and disease information; SME, satisfaction of medication effectiveness; IMS, impacts of medications and side-effects; PIMU, psychological impacts of medication use; CON, convenience; AA, availability and accessibility; TRHC, therapeutic relationships with healthcare providers; OQoL, overall QoL.

${ }^{*} p<0.01$.

${ }^{\dagger} p<0.05$.

Table 4. Multiple linear regression models of PROMPT-QoL domain scores

\begin{tabular}{|c|c|c|c|c|c|c|c|c|}
\hline Patient characteristic (independent variable) & MDI & SME & IMS & PIMU & CON & AA & TRHC* & OQoL \\
\hline Age (years) & $-0.201^{\dagger}$ & 0.132 & 0.086 & 0.038 & $0.176^{\ddagger}$ & $0.204^{\dagger}$ & - & $0.187^{\dagger}$ \\
\hline Gender (1, female; 2, male) & -0.042 & 0.020 & 0.102 & $0.145^{\ddagger}$ & 0.067 & 0.047 & - & 0.010 \\
\hline Number of education years & -0.011 & 0.003 & 0.066 & -0.001 & -0.011 & -0.091 & - & -0.012 \\
\hline $\begin{array}{l}\text { Employment status } \\
\text { (1, unemployed/housewife/student/retired; } \\
\text { 2, employed) }\end{array}$ & 0.010 & -0.065 & 0.101 & 0.038 & -0.054 & -0.050 & - & -0.080 \\
\hline Duration of epilepsy (years) & -0.108 & 0.067 & 0.097 & $0.173^{\star}$ & -0.105 & 0.043 & - & 0.036 \\
\hline Number of antiepileptic drugs & 0.140 & 0.002 & -0.039 & 0.076 & 0.073 & 0.093 & - & 0.099 \\
\hline $\begin{array}{l}\text { Seizure frequency per three months } \\
(1,0 ; 2,>0)\end{array}$ & 0.067 & $-0.225^{\dagger}$ & -0.100 & 0.098 & $-0.183^{\dagger}$ & -0.070 & - & $-0.227^{\dagger}$ \\
\hline $\begin{array}{l}\text { Treatment preference ( } 1 \text {, medications; } 2 \text {, both } \\
\text { medications and alternative medications, } \\
\text { alternative medications, others) }\end{array}$ & -0.122 & $-0.245^{\dagger}$ & $-0.246^{\dagger}$ & $-0.233^{\dagger}$ & -0.044 & $-0.156^{\ddagger}$ & - & $-0.255^{\dagger}$ \\
\hline Adjusted $R^{2}$ & 0.035 & 0.115 & 0.055 & 0.092 & 0.059 & 0.054 & - & 0.157 \\
\hline
\end{tabular}

Values in the table indicate standardized regression coefficients.

PROMPT-QoL, Patient-Reported Outcomes Measure of Pharmaceutical Therapy for Quality of Life; MDI, medication and disease information; SME, satisfaction of medication effectiveness; IMS, impacts of medications and side-effects; PIMU, psychological impacts of medication use; CON, convenience; AA, availability and accessibility; TRHC, therapeutic relationships with healthcare providers; OQoL, overall QoL.

*No variables were entered into the model since there were no significant variables.

${ }^{\dagger} p<0.01$.

${ }^{\ddagger} p<0.05$.

variate and multivariate analyses). For univariate analysis, longer duration of epilepsy, free seizure and medication preference were significantly related with higher psychological impacts of medication use domain scores (Table 3). Nevertheless, the multivariate regression showed that male gender, longer duration of seizure, and medication preference were significantly correlated with higher psychological impacts of medication use domain scores (Table 4). Older age and free seizure were significantly associated with higher convenience domain scores (both univariate and multivariate analyses). Older age and medication preference were significantly correlated 
with higher availability and accessibility domain scores (both univariate and multivariate analyses). Univariate analyses found that therapeutic relationships with healthcare providers domain scores were not significantly related with all eight patient characteristics (Table 3). Hence, there were no variables entered into the stepwise multivariate linear regression (Table 4). Older age, free seizure, and medication preference were significantly associated with higher overall quality of life domain scores (both univariate and multivariate analyses).

\section{Discussion}

To the best of our knowledge, this is the first study to extensively assess medication therapy-related quality of life using a new specific medication questionnaire (i.e., PROMPT-QoL) and its associations with patients' demographic and clinical characteristics in epilepsy patients.

\section{Medication-related quality of life}

For the general attitude toward medication use item, unsurprisingly, most patients preferred to use only medications for treating their epilepsy since the original purpose of their visit to the hospital was to receive antiepileptic drugs. However, approximately $19 \%$ of the sample wanted the combination between medication, alternative treatments and only alternative treatments. This is in line with the study conducted in Thailand reporting that $12.5 \%$ of patients with epilepsy used herbal medication use. ${ }^{18}$

For the other eight domains, the Thai patients with epilepsy provided seven mean domain scores between 61.9 and 72.8, which were considered as moderate-to-good. Only therapeutic relationships with health care providers domain had the highest mean domain score of 77.9, meaning a good-to-excellent medication therapy-related quality of life.

Among the eight domains, the psychological impacts of medication use domain yielded the lowest score of 61.9. The reason that patients with epilepsy had the lowest domain score for the psychological impacts of medication use may be due to the fact that most of them were still young (the mean age of 36). Hence, they were feared or worried about medication side effects, dependence, taking many medications, and their use of medication making them less healthy than person with the same age since these items provided low scores $(<3.5)$. This is in line with a previous study that found patients with drug refractory epilepsy were worried about the long-term side ef- fects of the drugs on their body. ${ }^{12}$ Additionally, they were concerned about changing type/strength of medication, medication ineffectiveness, and feeling bored with taking medication every day (item scores of 3.5). Therefore, healthcare providers should take more care of these concerns of patients to enhance their medication therapy-related quality of life.

In addition to the psychological impacts of medication use items above, the items with low scores $(\leq 3.5)$ of their domains indicated that Thai healthcare providers should pay more attention. They included "strength", "what to do if medication doses are missed", the impacts of medications' side-effects on memory and cognition", "appropriate dosage forms", "service process and waiting time", and "travel or self-support to hospital."

\section{Associations between medication-related quality of life and patient characteristics}

Both univariate and multivariate analyses found that age and treatment preference were significantly associated with most PROMPT-QoL domains. The older patients tended to have higher domain scores except for the medication and disease Information domain. A possible explanation is that the younger patients were more likely to ask healthcare providers the questions about drug and disease information when they did not understand than the older patients. However, the younger patients had lower scores of the convenience, availability and accessibility, and overall quality of life domains, which healthcare providers should pay more attention to. The patients who preferred only medication treatment also tended to have higher PROMPT-QoL domain scores. This is consistent with a previous study in Thai patients with chronic diseases. ${ }^{10}$ A possible reason is that the patients who were not satisfied and worried about taking their medications were more likely to seek alternative medications or other treatments that those who were and were not.

The univariate analysis showed that the patients with at least one seizure in 3 months had significantly lower four domain scores, but the multivariate analysis found three significant domains. These results are in line with previous studies which found that good seizure control was significantly related with higher health-related quality of life. ${ }^{4-6} \mathrm{~A}$ possible reason is that the patients with more seizure frequencies were more likely to be unsatisfied and inconvenient about taking antiepileptic drugs than those free from seizure.

Based on univariate and multivariate analyses, education and employment status were not significantly associated with all 8 PROMPT-QoL domain scores. This result is consistent with a previous 
study which reported that education and employment status did not significantly affect health-related quality of life. ${ }^{13}$ Additionally, gender was not significantly related with PROMPT-QoL domain scores in the univariate analyses. However, the multivariate analysis found that females were more likely to have lower psychological impacts of medication use than males. This contrasts with a previous systemic review that found that gender was not significantly associated with health-related quality of life in adults with epilepsy. ${ }^{15}$

Higher number of antiepileptic drugs was significantly associated with higher medication and disease information domain score in the univariate analysis. However, after adjusting for covariates, no significant result was found. This is in line with a previous study reporting that number of antiepileptic drugs was not significantly associated with health-related quality of life in Iraq patients with epilepsy. ${ }^{19}$ Moreover, duration of epilepsy was significantly correlated with the medication and disease information and psychological impacts of medication use domain scores in the univariate analysis. Nevertheless, the multivariate analysis found that the patients with longer duration of seizure had significantly higher psychological impacts of medication use domain score than those with shorter duration. This is not consistent with a previous study which reported that lower health-related quality of life was significantly associated with longer duration of the disease. ${ }^{13-14} \mathrm{~A}$ possible explanation is that the new patients might not get used to taking antiepileptic drugs, so they were more worried or concerned about taking them.

\section{Strengths and limitations}

This study comprehensively assessed medication-related quality of life and its associations with patient characteristics in epilepsy patients, which have not been reported before. Nevertheless, it had some limitations as follows. First, since this study was conducted in only one public university hospital in Bangkok, the capital city of Thailand, it might not be generalized to other settings. Second, this study also employed convenience sampling, so it might cause sampling bias. Hence, further research can be done in other healthcare settings and apply a random sampling method.

This study has demonstrated the medication-related quality of life of Thai patients with epilepsy using the PROMPT-QoL a novel specific medication measure for health-related quality of life. Most domain scores were moderate-to-good. Age, gender, duration of epilepsy, seizure frequency, and treatment preference were significantly associated with PROMPT-QoL domain scores in multivariate linear regression analyses. Healthcare providers should pay more attention to patients' psychological impacts of antiepileptic drugs and those with characteristics related to lower medication-related quality of life.

\section{Acknowledgements}

This study was funded by Faculty of Pharmaceutical Sciences, Chulalongkorn University. The authors thank Pichtanun Tiebhieng, Wanutchaya Keremek, and Siriporn Mekpayub for their data collection and all patients for their participation in the study and the hospital staff for assistance with the data collection.

\section{Conflict of Interest}

The authors declare that they have no conflicts of interest.

\section{References}

1. World Health Organization (WHO). Epilepsy [Internet]. Genever: WHO, [cited 2019 Aug 21]. Available at : https://www.who.int/news-room/ fact-sheets/detail/epilepsy.

2. Asawavichienjinda T, Sitthi-Amorn C, Tanyanont W. Prevalence of epilepsy in rural Thailand: a population-based study. J Med Assoc Thai 2002;85: 1066-73.

3. Rogers SJ, Cavazos JE. Epilepsy. In: Dipiro JT, Talbert RL, Yee GC, Matzke GR, Wells BG, Posey ML, eds. Pharmacotherapy: a pathophysiologic approach. 8th ed. New York: McGraw-Hill, 2011;979.

4. Santos AMC, Castro-Lima H, Matos MAA, Brito MB. Quality of life among women with epilepsy during their reproductive years. Epilepsy Behav 2018;85:10-3.

5. Chen HF, Tsai YF, His MS, Chen JC. Factors affect quality of life in adults with epilepsy in Taiwan: a cross-sectional, correlational study. Epilepsy Behav 2016;58:26-32.

6. Blond BN, Detyniecki K, Hirsch L. Assessment of treatment side effects and quality of life in people with epilepsy. Neuro Clin 2016;34:395-410, viii.

7. Devinsky O, Vickrey BG, Cramer J, et al. Development of the quality of life in epilepsy inventory. Epilepsia 1995;36:1085-104.

8. Sakthong $P$, Suksanga $P$, Sakulbumrungsil $R$, Winit-Watjana W. Development of patient-reported outcomes measure of pharmaceutical therapy for quality of life (PROMPT-QoL): a novel instrument for medication management. Res Soc Admin Pharm 2015;11:315-38.

9. Cipolle RJ, Strand LM, Morley PC. Pharmaceutical care practice: the clinician's guide. 2nd ed. New York: McGraw-Hill, 2004.

10. Sakthong P, Chinthammit C, Sukarnjanaset P, Sonsa-Ardjit N, Munpan $W$. Psychometric properties of the patient-reported outcomes measure of pharmaceutical therapy for quality of life (PROMPT-QoL). Value Health Reg Issues 2017;12:41-9.

11. Sakthong P, Sangthonganotai T. A randomized controlled trial of the impact of pharmacist-led patient-centered pharmaceutical care on pa- 
tients' medicine therapy-related quality of life. Res Soc Admin Pharm 2018;14:332-39.

12. Puri I, Dash D, Padma MV Tripathi M. Quality of life and its determinants in adult drug refractory epilepsy patients who were not candidates for epilepsy surgery: a correlational study. J Epilepsy Res 2018;8:81-6.

13. Melikyan E, Guekht A, Milchakova L, Lebedeva A, Bondareva I, Gusev E. Health-related quality of life in Russian adults with epilepsy: the effect of socio-demographic and clinical factors. Epilepsy Behav 2012;25: 670-5.

14. Edefonti V, Bravi $F$, Turner $K$, et al. Health-related quality of life in adults with epilepsy: the effect of age, age at onset and duration of epilepsy in a multicentre Italian study. BMC Neurol 2011;11:33.

15. Taylor RS, Sander YW, Taylor RJ, Baker GA. Predictors of health-related quality of life and costs in adults with epilepsy: a systematic review. Epilepsia 2011;52:2168-80.

16. Nunnally JC Jr. Psychometric Theory. 2nd ed. New York: McGraw-Hill, 1978.

17. Neter J, Kutner MH, Nachtsheim CJ, Wasserman W. Applied Linear Statistical Models. 4nd ed. New York: McGrow-Hill, 1996.

18. Peltzer $K$, Pengpid $S$. The use of herbal medicines among chronic disease patients in Thailand: a cross-sectional survey. I Multidiscip Healthc 2019;12:573-82.

19. Shakir M, Al-Asadi JN. Quality of life and its determinants in people with epilepsy in Basrah, Iraq. Sultan Qaboos Univ Med J 2012;12: 449-57. 